\title{
Near Room Temperature Magnetocaloric Response of an (FeNi)ZrB Alloy
}

\author{
Jhon J. Ipus, Huseyin Ucar, and Michael E. McHenry \\ Department of Materials Science and Engineering, Carnegie Mellon University, Pittsburgh, PA 15213 USA
}

\begin{abstract}
Mechanical alloying of $\left(\mathrm{Fe}_{70} \mathrm{Ni}_{30}\right)_{89} \mathrm{Zr}_{7} \mathrm{~B}_{4}$ powders leads to the formation of a $\gamma$-FeNi phase. In order to obtain single $\gamma$-phase, a powdered sample was solution annealed in the $\gamma$-phase field and water quenched. The Curie temperature of this powder was slightly higher than room temperature. The refrigerant capacity calculated for this alloy, $R_{\mathrm{FWHM}} \sim 330 \mathrm{~J} \cdot \mathrm{kg}^{-1}$ for $5 \mathrm{~T}$, is comparable to other prominent room temperature magnetocaloric materials, making this alloy a good candidate for magnetic refrigeration near room temperature with additional benefits that is non-rare earth containing and less expensive than many alternatives.
\end{abstract}

Index Terms-Ball milling, iron alloys, magnetic materials, magnetic properties, nanostructured materials.

\section{INTRODUCTION}

$\mathbf{T}$ HE magnetocaloric effect is a property of any magnetic material and is defined as the heating or cooling of a magnetic material as a response of the application of a magnetic field. Magnetocaloric materials are subject of current interest due to the promising application of magnetic refrigeration around room temperature, because this technology is energetically more efficient than that based on conventional gas compression refrigeration, about $20 \%$ [1], and it is more environmental friendly because ozone depleting and warming global refrigerants are not used.

Rare earths metals, as Gd, and their compounds have been extensively studied in the last years because these materials present a large magnetocaloric response and their work temperatures are close to room temperature [2], [3]. However, the high price of Gd and the proclivity to corrosion of these compounds have prevented their use for commercial purposes. In order to reduce the cost of material production and due to a desire to limit the necessity for rare-earth elements with limited abundances, transition-metal-based alloys are being investigated to use instead rare-earth-based materials [4].

In order to produce these materials, mechanical alloying has become a very versatile technique used for direct production of metastable microstructures (amorphous, nanocrystalline, supersaturated solid solutions, etc.) from elemental powders or alloys [5], [6]. During milling process, the material is submitted to fracture and cold welding, as well as intensive plastic deformation, which define the powder morphology, microstructure, and properties. The continuous storing of defects in the crystalline phases during milling process destabilizes them, leading to creation of nanocrystalline and/or amorphous structures [6].

In amorphous precursors to nanocomposites magnets, it is often possible to suppress formation of stable phase and to, therefore, take advantage of properties of metastable phase, as has been shown recently for $\mathrm{Co}-\mathrm{Fe}$ nanocomposites [7]. In $\mathrm{Fe}-\mathrm{Ni}$-based nanocomposites, a similar phenomenon is observed in Fe-rich alloys [8], [9] where nucleation of the $\alpha$-phase is suppressed in favor of a metastable $\gamma$-phase that has a Curie

Manuscript received February 17, 2011; revised April 26, 2011 and May 25 , 2011; accepted June 09, 2011. Date of current version September 23, 2011. Corresponding author: M. E. McHenry (e-mail:mm7g@andrew.cmu.edu).

Color versions of one or more of the figures in this paper are available online at http://ieeexplore.ieee.org.

Digital Object Identifier 10.1109/TMAG.2011.2159781 temperature with a strong compositional dependence. This can also have profound effects on technical magnetic properties because on the Fe-rich side of the $\mathrm{Fe}-\mathrm{Ni}$ phase diagram there is a strong compositional dependence of the Curie temperature $T_{C}$ on composition in the $\gamma$-phase [10]. The tuning of the $T_{C}$ can impact the design of efficient magnetocaloric refrigerants. In this paper, initially we describe the formation of a metastable $\gamma$-FeNi phase by mechanical alloying from constituent powders, $\mathrm{Fe}-\mathrm{Ni}-\mathrm{Zr}-\mathrm{B}$, with composition similar to those produced by rapid solidification processing. After this, the stabilization of $\gamma$-phase, magnetic properties, and the magnetocaloric response of mechanically alloyed sample for larger time are presented.

\section{EXPERIMENTAL}

Nanoperm $\left(\mathrm{Fe}_{70} \mathrm{Ni}_{30}\right)_{89} \mathrm{Zr}_{7} \mathrm{~B}_{4}$ type alloy was produce by ball milling in a shaker mill (Spex 8000D) from elemental Fe, $\mathrm{Ni}, \mathrm{Zr}$, and prealloyed $\mathrm{Zr}_{2} \mathrm{~B}$ powders under $\mathrm{Ar}$ atmosphere. The initial powder mass was $7 \mathrm{~g}$ and the ball to powder ratio was 10:1. After selected, milling times powder samples were taken out from the vials to characterize crystal structure by $\mathrm{X}$-ray diffraction using $\mathrm{Cu} \mathrm{K} \alpha$ radiation in a X'Pert PRO MPD diffractometer.

In order to obtain a material with a single $\gamma$-FeNi phase, the sample milled up to $50 \mathrm{~h}$ was subsequently sealed in a quartz crucible with $\mathrm{Ar}$ atmosphere and annealed in the $\gamma$-phase region, $700{ }^{\circ} \mathrm{C}$, and quenched in water to stabilize the metastable $\gamma$-FeNi phase.

Magnetic properties were studied using a Lakeshore 7407 vibrating sample magnetometer using a maximum applied field of $0.5 \mathrm{~T}$ at constant temperatures in the range of room temperature to $553 \mathrm{~K}$

The magnetic entropy change due to the application of a magnetic field has been calculated using a numerical approximation to the equation

$$
\Delta S_{M}=\int_{0}^{H_{\text {max }}}\left(\frac{\partial M}{\partial T}\right)_{H} d H
$$

where $\Delta S_{M}$ is the magnetic entropy change, $M$ is the magnetization, and $T$ is the temperature. The partial derivative is replaced by finite differences and the integration is performed numerically from zero to the maximum value of the applied magnetic field $H_{\max }$. 


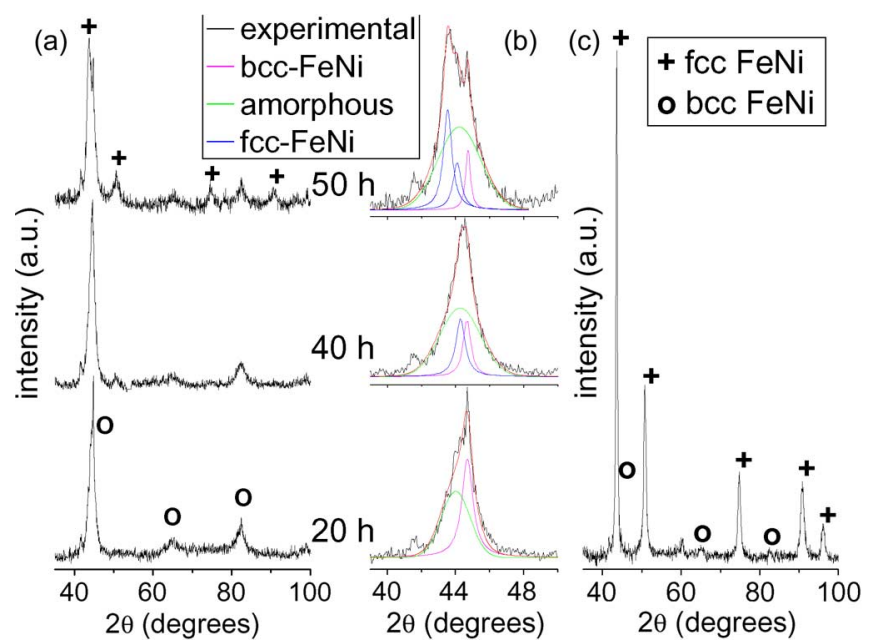

Fig. 1. (a) X-ray diffraction patterns of as-milled samples after different milling times. (b) Deconvolution of the main diffraction peak using different crystalline and amorphous contributions. (c) X-ray diffraction pattern of the sample milled $50 \mathrm{~h}$ after quenching from $\gamma$-phase region.

\section{RESUltS AND DiscuSSIONS}

X-ray diffraction patterns of powder samples after several milling times are presented in Fig. 1(a). It can be seen that besides the diffraction peaks corresponding to body-centered cubic (bcc)-FeNiZrB and face-centered cubic (fcc)-FeNiZrB phases, other small peaks are observed that can be indexed to an FeNi spinel oxide.

After the formation of a supersaturated bcc-FeNiZrB solid solution at short times, $t<20 \mathrm{~h}$, the presence of an amorphous phase is observed after $20 \mathrm{~h}$ milling along with the solid solution. This amorphous phase is attributed to the continuous accumulation of defects in the crystalline phase during milling process which destabilizes it, leading to an amorphous phase structure [6].

For longer milling times, the amorphous phase fraction is reduced and the formation of the fcc $\gamma$-FeNiZrB phase is observed as shown in Fig. 1(b) for $40 \mathrm{~h}$ milling time. The best fit to the experimental data was achieved by assuming two crystalline contributions to represent the (110) and (111) diffraction peaks of the $\alpha$-FeNiZrB and $\gamma$-FeNiZrB phases, respectively, and an amorphous halo.

After $50 \mathrm{~h}$ milling, the $\gamma$-FeNiZrB fraction has increased and the (111) fcc diffraction peak is clearly distinguished from the (110) bcc peak. However, a large broadening of the (111) fcc diffraction peak is observed that could be ascribed to a small crystal size (Scherrer broadening [11]) and/or a large amount of microstrain. The presence of crystallites with a range of composition is also possible, since from mechanical alloying, a distribution of the powder particle compositions is expected [12]. Fig. 1(b) shows the fit corresponding to sample milled $50 \mathrm{~h}$ where two crystalline contributions were necessary to simulate the (111) fcc diffraction peak, which indicate the presence of crystals with different composition into this phase.

Lattice parameter and crystal size values were obtained from $2 \theta$ position and full-width at half-maximum (FWHM) of the (110) bec and (111) fcc diffraction peaks. In this way, we have
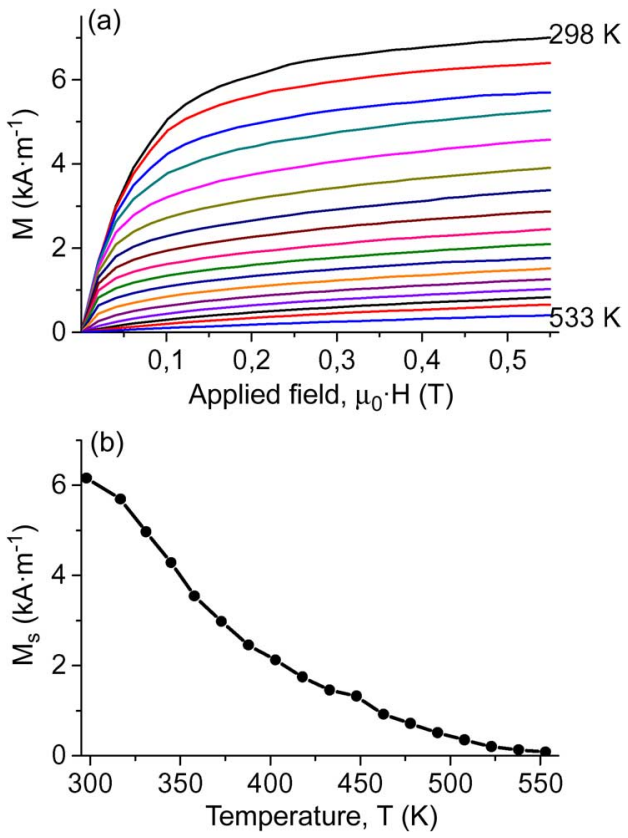

Fig. 2. (a) Magnetization $M$ isotherm curves obtained from room temperature to $533 \mathrm{~K}$ for a maximum applied field of $0.5 \mathrm{~T}$. (b) Temperature dependence of spontaneous magnetization $M_{S}$ for quenched powder sample.

calculated a minimum crystal size considering this as the only effect responsible for broadening of the XRD peak [12]. The lattice parameter of the $\alpha$-FeNiZrB phase has a nearly constant value of $a=2.867 \pm 0.003 \AA$ for the three milling times studied. This value is slightly higher than a literature value for a composition with the same nominal $\mathrm{Fe} / \mathrm{Ni}$ ratio [13]. This can be explained by some incorporation of $\mathrm{Zr}$ and $\mathrm{B}$ atoms in the bcc lattice. The crystal size, calculated using Scherrer formula, has a value of $D \sim 40 \mathrm{~nm}$ after $20 \mathrm{~h}$ milling and for larger milling times a minimum value of $D \sim 20 \mathrm{~nm}$ nm was found.

For the $\gamma$-FeNiZrB phase, the lattice parameter after $40 \mathrm{~h}$ milling present a value $a=3.543 \pm 0.004 \AA$ in agreement with the value reported in the literature [13]. For $50 \mathrm{~h}$ milling, two lattice parameters were calculated from the fit of the X-ray pattern, $a_{1}=3.556 \pm 0.004 \AA$ and $a_{2}=3.599 \pm 0.005 \AA$. The first of these values agrees with that found in [13] for $\gamma$-FeNi phase with 30 at. $\% \mathrm{Ni}$; the second value is larger than the previous one that could be ascribed to some dissolution of $\mathrm{Zr}$ (substitutional) and B (interstitial) atoms [14] in the fcc structure. The crystal size calculated by Scherrer analysis for this phase has a value of $D \sim 15 \mathrm{~nm}$ for all studied times.

In order to remove this phase distribution, sample milled up to $50 \mathrm{~h}$ was annealed in the $\gamma$-phase field and quenched. X-ray diffraction was performed to observe the structural development as illustrated in Fig. 1(c). It is seen that after this solution annealing treatment, the compositional distribution observed in the original as-milled sample is no longer detected. The crystalline $\gamma$-FeNiZrB phase has a lattice parameter $a=$ $3.596 \pm 0.004 \AA$ and a crystal size $D \sim 20 \mathrm{~nm}$. A small trace of $\alpha$-FeNiZrB phase was also observed.

For quenched sample, isothermal magnetization curves for a maximum applied field of $0.5 \mathrm{~T}$ were taken every $15 \mathrm{~K}$ in a temperature range of room temperature to $553 \mathrm{~K}$ as shown 

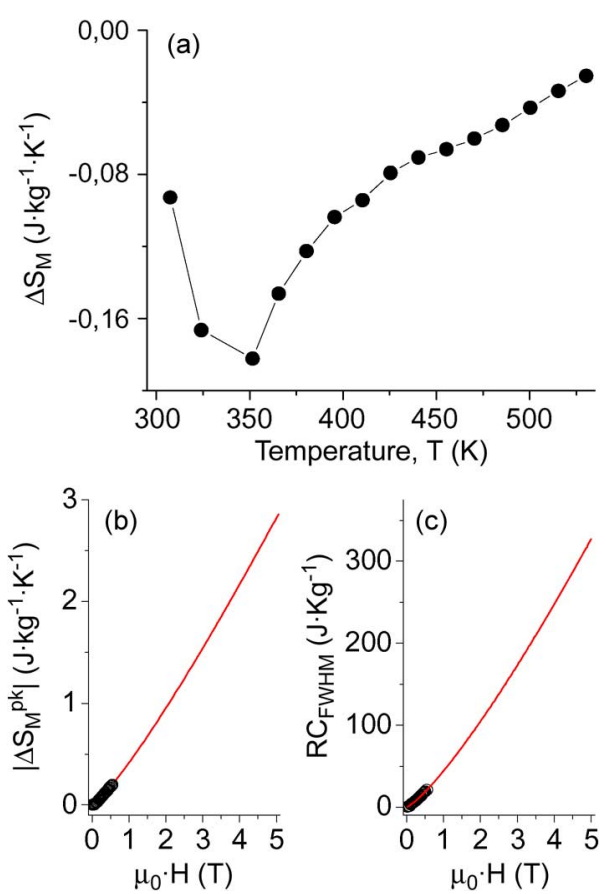

Fig. 3. (a) Magnetic entropy change $\Delta S_{M}$ as a function of temperature of quenched sample for a maximum applied field of $0.5 \mathrm{~T}$. (b) Field dependence of the peak magnetic entropy change $\Delta S_{M}^{\mathrm{pk}}$. (c) Field dependence of the refrigeration capacity, $\mathrm{RC}_{\mathrm{FWHM}}$. Symbols correspond to experimental data and line represents the extrapolated values from the power law.

in Fig. 2(a). It can be seen a continuous decrease in magnetization as the measuring temperature increases due to the ferromagnetic to paramagnetic transformation. It is also observed that for high-applied magnetic field, magnetization values reflect a nonsaturated behavior. This could be explained by an unsaturated sample for these applied magnetic fields or by a superparamagnetic behavior of the powder particles. In this case, the second way was taken into account for the powder sample since this behavior is still observable for higher applied magnetic fields. From these isothermal curves, spontaneous magnetization values were obtained as the extrapolation to zero field of a linear fitting of the high-field magnetization.

Fig. 2(b) shows the spontaneous magnetization $M_{S}$ as a function of temperature for quenched powder. It can be seen that the ferromagnetic to paramagnetic transformation corresponding to the $\gamma$-FeNiZrB phase, $T_{C} \sim 342 \mathrm{~K}$, takes place at a slightly higher temperature than room temperature. This value calculated using the inflexion point method is in good agreement with the estimated values obtained from the compositional dependence of $T_{C}$ with Ni content for $\gamma$-Fe-Ni phase [13]. The long tail observed at higher temperatures keeps the magnetization from falling to zero. This could be ascribed to the trace amounts of an $\alpha$-FeNiZrB phase detected by X-ray diffraction, which has a higher Curie temperature than $\gamma$-FeNiZrB phase.

The magnetocaloric response was calculated from isothermal magnetization curves. Fig. 3(a) illustrates the magnetic entropy change for the studied alloy for a maximum applied magnetic field of $0.5 \mathrm{~T}$. The maximum magnetic entropy change value observed for the actual system is $0.182 \mathrm{~J} \cdot \mathrm{kg}^{-1} \mathrm{~K}^{-1}$. Moreover, the peak is very close to the Curie temperature which is expected for materials that present second-order magnetic phase transition [15].

The magnetic entropy change can be expressed as a power law of the magnetic field for materials with second-order phase transition [16]

$$
\Delta S_{M}^{\mathrm{pk}}=A H^{n}
$$

where $\mathrm{A}$ is a prefactor. Taking into account this power law, the peak entropy change value is extrapolated to larger magnetic fields [see Fig. 3(b)] to yield a value of $\sim 2.8 \mathrm{~J} \cdot \mathrm{kg}^{-1} \mathrm{~K}^{-1}$ for a maximum applied magnetic field of $5 \mathrm{~T}$.

Other parameter useful to characterize the magnetocaloric effect is the refrigerant capacity. In the present case, the refrigerant capacity was calculated from the FWHM times the absolute value of the peak entropy change value $\mathrm{RC}_{\mathrm{FWHM}}$, obtaining a value of $\sim 20 \mathrm{~J} \cdot \mathrm{kg}$ for $0.5 \mathrm{~T}$. $\mathrm{RC}_{\mathrm{FWHM}}$ can also be represented as a power law of the applied magnetic field [17], and a value of $\sim 330 \mathrm{~J} \cdot \mathrm{kg}^{-1}$ is obtained for a maximum applied field of $5 \mathrm{~T}$ as is shown in Fig. 3(c). This estimated value of $\mathrm{RC}_{\mathrm{FWHM}}$ is of the same order than those found in the literature for mechanical alloyed $\mathrm{Pr}_{2} \mathrm{Fe}_{17}$ alloy [18], $\mathrm{Gd}_{5} \mathrm{Ge}_{2-X} \mathrm{Si}_{2} \mathrm{Fe}_{X}$ prepared by arc-melting [3], $\mathrm{MnFeP}_{1-X} \mathrm{As}_{X}$ compound [19], ballmilled $\mathrm{Mn}-\mathrm{Fe}-\mathrm{P}-\mathrm{Ge}$ alloy [20] and melt spun $\mathrm{Fe}-\mathrm{B}-\mathrm{Cr}-\mathrm{Gd}$ alloy [21], which are considered as good materials for magnetic refrigeration near room temperature.

Hence, although the peak entropy change value is not large, the studied composition can be considered as a good candidate for magnetic refrigeration around room temperature, since the refrigerant capacity is the best parameter to describe the magnetocaloric effect at high temperatures, like room temperature.

\section{CONCLUSION}

Microstructure and magnetocaloric response of mechanically alloyed $\left(\mathrm{Fe}_{70} \mathrm{Ni}_{30}\right)_{89} \mathrm{Zr}_{7} \mathrm{~B}_{4}$ composition have been studied.

At short milling times, a bcc-FeNiZrB supersaturate solid solution is formed in the powders and after $40 \mathrm{~h}$ milling, a metastable $\gamma$-FeNiZrB phase was developed in the system with a crystal size in the order of tens nanometer. The crystalline $\gamma$-FeNiZrB phase presented a compositional distribution at the end of the studied milling time.

To obtain a single $\gamma$-FeNiZrB phase, the composition was heated in the $\gamma$-phase region and immediately water quenched. After this quenching, the compositional distribution previously observed is no longer detected and the crystal size remains of the same order.

Curie temperature and peak entropy change temperature are slightly larger that room temperature for this composition. The peak magnetic entropy change value is small but its refrigerant capacity is of the same order than materials that present giant magnetocaloric effect. Therefore, this alloy is suggested as a promising material for application in magnetic refrigeration near room temperature.

\section{ACKNOWLEDGMENT}

J. J. Ipus and M. E. McHenry acknowledge the support from Small Business Technology Transfer Program Proposal "Novel Management of Transducer Heat and Nonlinearity" Topic No.: N08-T020 under Contract No. N00014-08-M-0313. J. J. Ipus 
acknowledges a Postdoctoral Research Associate position from Carnegie Mellon University, Pittsburgh, PA.

\section{REFERENCES}

[1] K. A. Gschneidner, Jr. and V. K. Pecharsky, "Magnetocaloric materials," Annu. Rev. Mater. Sci., vol. 30, pp. 387-429, 2000.

[2] V. K. Pecharsky and K. A. Gschneidner, Jr., "Giant magnetocaloric effect in $\mathrm{Gd}_{5}\left(\mathrm{Ge}_{2} \mathrm{Si}_{2}\right)$," Phys. Rev. Lett., vol. 78, pp. 4494-4497, 1997.

[3] V. Provenzano, A. J. Shapiro, and R. D. Shull, "Reduction of hysteresis losses in the magnetic refrigerant $\mathrm{Gd}_{5} \mathrm{Ge}_{2} \mathrm{Si}_{2}$ by the addition of iron," Nature, vol. 429, pp. 853-857, 2004.

[4] O. Tegus, E. Brück, K. H. J. Buschow, and R. F. de Boer, "Transitionmetal-based magnetic refrigerants for room-temperature applications," Nature, vol. 415, pp. 150-152, 2002.

[5] M. E. McHenry, M. A. Willard, and D. E. Laughlin, "Amorphous and nanocrystalline materials for application as soft magnets," Prog. Mater. Sci., vol. 44, pp. 291-433, 1999.

[6] C. Suryanarayana, "Mechanical alloying and milling," Prog. Mater. Sci., vol. 46, pp. 1-184, 2001.

[7] P. R. Ohodnicki, Y. L. Qin, D. E. Laughlin, M. E. McHenry, M. Kodzuka, T. Ohkubo, K. Hono, and M. A. Willard, "Composition and non-equilibrium crystallization in partially devitrified Co-rich soft magnetic nanocomposite alloys," Acta Mater., vol. 57, pp. 87-96, 2009.

[8] A. L. Greer and I. T. Whitaker, "Transformations in primary crystallites in (Fe,Ni)-based metallic glasses," Mater. Sci. Forum, vol. 386-388, pp. 77-88, 2002.

[9] K. J. Miller, M. Sofman, K. McNerny, and M. E. McHenry, "Metastable $\gamma$-FeNi nanostructures with a tunable curie temperatures," J. Appl. Phys. [Dig. 11th Joint MMM-INTERMAG Conf., 2010], vol. 107, p. 09A305, 2010.

[10] K. B. Reuter, D. B. Williams, and J. I. Goldstein, "Determination of the Fe-Ni phase diagram below $400{ }^{\circ}$ C," Metall. Mater. Trans. A, vol. 20, pp. 719-725, 1989.

[11] M. DeGraef and M. E. McHenry, Structure of Materials. Cambridge, U.K.: Cambridge Univ. Press, 2007 [Online]. Available: http://som. web.cmu.edu
[12] J. J. Ipus, J. S. Blázquez, A. Conde, M. Krasnowski, and T. Kulik, "Analysis of the mechanically alloyed Fe85Nb5B10 powder using a non-unique lattice parameter," J. Non-Cryst. Solids [Dig. 9th Workshop on Non-Cryst. Solids, 2008], vol. 354, pp. 5132-5134, 2008.

[13] L. J. Swartzendruber, V. P. Itkin, and C. B. Alcock, "The Fe-Ni (ironnickel) system,” J. Phase Equilibria, vol. 12, pp. 288-311, 1991.

[14] C. Y. Um, F. Johnson, M. Simone, J. Barrow, and M. E. McHenry, "Effect of crystal fraction on hardness in FINEMET and NANOPERM nanocomposite alloys," J. Appl. Phys. [Dig. 49th Annu. Conf. Magn. Magn. Mater., 2004], vol. 97, p. 10F504, 2005.

[15] V. Franco, A. Conde, M. D. Kuzmin, and J. M. Romero-Enrique, "The magnetocaloric effect in materials with a second order phase transition: Are $\mathrm{T}_{\mathrm{C}}$ and $\mathrm{T}_{\text {peak }}$ necessarily coincident?," J. Appl. Phys. [Dig. 53rd Annu. Conf. Magn. Magn. Mater., 2008], vol. 105, p. 07A917, 2009.

[16] V. Franco, J. S. Blázquez, and A. Conde, "Field dependence of the magnetocaloric effect in materials with a second order phase transition: A master curve for the magnetic entropy change," Appl. Phys. Lett., vol. 89 , p. 222512, 2006.

[17] V. Franco, A. Conde, J. M. Romero-Enrique, and J. S. Blázquez, "A universal curve for the magnetocaloric effect: An analysis based on scaling relations," J. Phys.: Condens. Mater., vol. 20, p. 285207 , 2008.

[18] P. Gorría, J. L. Sánchez-Lamazares, P. Alvarez, M. J. Pérez, J. Sanchez-Marcos, and J. A. Blanco, "Relative cooling power enhancement in magneto-caloric nanostructure Pr2Fe17," J. Phys. D: Appl. Phys., vol. 41, p. 192003, 2008.

[19] E. Brück, J. Kamarad, V. Sechovsky, Z. Arnold, O. Tegus, and F. R. de Boer, "Pressure effect on the magnetocaloric properties of $\mathrm{MnFeP}_{\mathrm{X}-1} \mathrm{As}_{\mathrm{X}}$," J. Magn. Magn. Mater., vol. 310, pp. E1008-E1009, 2007.

[20] W. Dagula, O. Tegus, B. Fuquan, L. Zhang, P. Z. Si, M. Zhang, W. S. Zhang, E. Brück, F. R. de Boer, and K. H. J. Buschow, "Magneto entropy change in $\mathrm{Mn}_{1.1} \mathrm{Fe}_{0.9} \mathrm{P}_{1-\mathrm{X}} \mathrm{Ge}_{X}$ compounds," IEEE Trans. Mag. [Dig. T-MAG, 2005], vol. 41, no. 10, pp. 2778-2780, Oct. 2005.

[21] J. Y. Law, R. V. Ramanujan, and V. Franco, "Tunable curie temperatures in Gd alloyed Fe-B-Cr magnetocaloric materials," J. Alloys Compounds, vol. 508, pp. 14-19, 2010. 